\title{
Noncommutative Brownian Motion in Monotone Fock Space
}

\author{
Naofumi Muraki \\ Supported by Grant-in-Aid for Scientific Research No. 07740157, the Ministry of Education, Science and \\ Culture, Japan
}

Received: 15 March 1996/ Accepted: 2 July 1996

Dedicated to Professor Minoru Motoo on his $70^{\text {th }}$ birthday

\begin{abstract}
An example of noncommutative Brownian motion is constructed on the monotone Fock space which is a kind of "Fock space" generated by all the decreasing finite sequences of positive real numbers. The probability distribution at time $t \geq 0$ associated to this Brownian motion is shown to be the arcsine law normalized to mean 0 and variance $t$.
\end{abstract}

\section{Introduction}

In quantum probability theory or noncommutative probability theory, several (noncommutative) Brownian motions have been known (see Parthasarathy [Par], Meyer [Mey], and Schürmann [Sch2]). For example, the following have been known.

The noncommuting pair of classical Brownian motions arises from the boson Fock space in which the commuting independence and the commuting central limit theorem are concerned (see Segal [Seg], Cushen and Hudson [CuH], Giri and von Waldenfels [GvW], and Hudson and Parthasarathy [HuP]). The associated probability law to this bosonic Brownian motion is the Gaussian distribution.

The noncommuting pair of fermion Brownian motions arises from the fermion Fock space in which the anticommuting independence and the anticommuting central limit theorem are concerned (see Hudson [Hud], von Waldenfels [vW], Barnett, Streater and Wilde [BSW], and Applebaum and Hudson [ApH]). The associated probability law to the fermion Brownian motion is the distribution of Bernoulli type. The noncommuting pair of free Brownian motions arises from the free Fock space in which the free independence and the free central limit theorem are concerned (see Voiculescu [Voi], Speicher [Spe], and Kümmerer and Speicher [KuS]). The associated probability law to the free Brownian motion is the Wigner semicircle law. There is also a one-parameter

\footnotetext{
* Supported by Grant-in-Aid for Scientific Research No. 07740157, the Ministry of Education, Science and Culture, Japan
} 
family of Brownian motions called the generalized Brownian motions which interpolate the three important Brownian motions of boson, fermion and free (see Bozejjkoand Speicher [BoS], and Schürmann [Sch1]). These noncommutative Brownian motions are deeply connected with the general notion of "independence" and "Fock space." In these examples, "Fock space" is used to realize in a concrete Hilbert space the abstract notion of noncommutative "independence" and the associated "Brownian motion (or white noise)." The general notion of "independence" and "white noise" in quantum probability theory was formulated by Kümmerer (see [Kum, Spe]).

In a previous paper [Mur1], for the purpose of constructing an example of the noncommutative "de Moivre-Laplace theorem," we introduced the (discrete time) monotone Fock space which is a kind of "Fock space" generated by all the decreasing finite sequences of natural numbers. We proved in this Fock space a noncommutative "de Moivre-Laplace theorem" where the limit distribution was shown to be, neither the Gaussian law nor the Wigner semicircle law, but the arcsine law normalized to mean 0 and variance 1 .

In this paper, for the purpose of constructing an example of noncommutative "Brownian motion," we introduce the (continuous time) monotone Fock space which is the natural generalization of previously introduced (discrete time) monotone Fock space to the continuous time situation. We construct on the monotone Fock space an example of noncommutative Brownian motion where the associated probability distribution at time $t$ is shown to be $\mu_{t}(d x)=\mu(d x / \sqrt{t})$. Here $\mu$ is the "standard arcsine law" obtained in the previous paper.

The paper is organized as follows. In Sect. 2, we give the definitions of the monotone Fock space, the canonical processes and related operators. In Sect. 3, we investigate the independence structure of monotone Fock space. It is proved that the canonical pair process on monotone Fock space is a process with independent increments in the Kümmerer independence. In Sect. 4, we calculate the probability distribution of the canonical process under the vacuum state. The probability distribution at $t \geq 0$ is shown to be the arcsine law with mean 0 and variance $t \geq 0$. The canonical processes are interpreted as noncommutative Brownian motions.

\section{Monotone Fock Space and Canonical Processes}

In this section, we give the definition of (continuous time) monotone Fock space. The von Neumann algebra with the vacuum state associated to the monotone Fock space is a quantum probability space where we construct a noncommutative Brownian motion. First we remark that our construction of monotone Fock space is analogous to the treatment of symmetric Fock space given in Maassen [Maa].

Let $T=\mathbb{R}_{+}$be the set of all positive real numbers $t \geq 0$. It is interpreted as the set of times. We make the convention that we use the short notation $\tau=\left(t_{1}>t_{2}>\right.$ $\left.\cdots>t_{r}\right)$ to mean an $r$-tuple $\left(t_{1}, t_{2}, \cdots, t_{r}\right)$ with $t_{i} \in T$ and $t_{1}>t_{2}>\cdots>t_{r}$. Let $\Sigma_{r}=\left\{\left(t_{1}>t_{2}>\cdots>t_{r}\right) \mid t_{i} \in T\right\}$ be the set of all decreasing finite sequences $\tau=\left(t_{1}>t_{2}>\cdots>t_{r}\right)$ of length $r$ from $T$. We assume that $\Sigma_{0}=\{\Lambda\}$ the singleton consisting of the null sequence $\Lambda$. For a pair $\sigma=\left(s_{1}>\cdots>s_{r}\right), \tau=\left(t_{1}>\cdots>t_{s}\right)$ of decreasing sequences satisfying $s_{r}>t_{1}$, denote by $(\sigma>\tau)$ a new decreasing sequence $\left(s_{1}>\cdots>s_{r}>t_{1}>\cdots>t_{s}\right)$ obtained by natural composition of $\sigma$ and $\tau$. We also assume that $(\sigma>\Lambda)=\sigma$ and $(\Lambda>\tau)=\tau$. Let $\mathcal{H}_{r}=L^{2}\left(\Sigma_{r}\right), r \geq 1$ be the complex Hilbert space of all $L^{2}$-functions on $\Sigma_{r}$ with respect to the Lebesgue measure $\lambda$ (induced from that of $\mathbb{R}^{r}$ ), which we call the r-particle space. The scalar product 
$<\psi\rangle$ is assumed to be linear in the right-hand side variable. The vacuum space (0-particle space) $\mathcal{H}_{0}$ is the $L^{2}$-space $L^{2}(\{\Lambda\})$ over the singleton $\{\Lambda\}$ with unit mass. We make the natural identification of $\mathcal{H}_{0}=L^{2}(\{\Lambda\})$ with the complex number field $\mathbb{C}$. Let $\Omega: \Lambda \mapsto 1$ be the vector of $\mathcal{H}_{0}$ corresponding to the unit element $1 \in \mathbb{C}$ in this identification. Let $\Phi=\oplus_{r} \mathcal{H}_{r}$ be the Hilbert space direct sum of $r$-particle spaces, which we call the (continuous time) monotone Fock space. The vector $\Omega$ as an element of monotone Fock space $\Phi$ is called the vacuum vector.

To make the description possibly simple, throughout this paper, we will not distinguish two $L^{2}$-integrable, measurable functions $f(\sigma)$ and $f_{1}(\sigma)$ if $f(\sigma)=f_{1}(\sigma)$, $\mu$-a.e. $\sigma$. So we omit the phrase " $\mu$-a.e. $\sigma$," whenever there is no need to emphasize the distinction with $\mu$-measure 0 between two measurable functions.

We define the monotone product $f \triangleright g$ of two vectors $f \in \mathcal{H}_{r}$ and $g \in \mathcal{H}_{s}$ by

$$
(f \triangleright g)(\sigma>\tau)=f(\sigma) g(\tau) .
$$

It is a vector in $\mathcal{H}_{r+s}$. Note that $f \triangleright g=0$ if $f, g \in \mathcal{H}_{1}$ and the support of $f$ is on the left-hand side of the support of $g$, i.e. $\forall s \in \operatorname{supp}(f), \forall t \in \operatorname{supp}(g), s<t$. This product naturally gives a structure of associative algebra to a dense subspace $\Phi_{0}=$ "the algebraic direct sum of $r$-partcle spaces" $\subset \Phi$. For each one-particle vector $h \in \mathcal{H}_{1}$, we define the creation operator $\delta_{h}^{+}$as the left multiplication operator with respect to the monotone product:

$$
\delta_{h}^{+} u=h \triangleright u, u \in \Phi_{0} .
$$

We write its bounded linear extension by the same symbol $\delta_{h}^{+}$. Note that $\delta_{h}^{+} \Omega=h$. We define the annihilation operator $\delta_{h}^{-}$as the adjoint of the creation operator. Its explicit action on an $r$-particle vector $u=u(\tau)=u\left(t_{1}>\cdots>t_{r}\right)$ with $r \geq 1$ is shown to be the integral operator with respect to the left-hand side variable:

$$
\left(\delta_{h}^{-} u\right)\left(t_{2}>\cdots>t_{r}\right)=\int_{t_{1}>t_{2}>\cdots>t_{r}} d t_{1} \overline{h\left(t_{1}\right)} u\left(t_{1}>t_{2}>\cdots>t_{r}\right) .
$$

Note that $\delta_{h}^{-} \Omega=0$. The map $h \mapsto \delta_{h}^{+}$is linear and the map $h \mapsto \delta_{h}^{-}$is antilinear.

Let $\mathcal{A}=W^{*}\left(1, \delta_{h}^{+}, \delta_{h}^{-} \mid h \in \mathcal{H}_{1}\right)$ be the von Neumann algebra generated by all the creation and annihilation operators with identity 1 . The von Neumann algebra $\mathcal{A}$ has a special state $\phi(\cdot)=\langle\Omega \mid \cdot \Omega\rangle$ called the vacuum state. The pair $(\mathcal{A}, \phi)$ is interpreted as a quantum probability space.

Denote by $\chi_{I}$ the indicator function of a finite interval $I \subset \mathbb{R}_{+}$, and let us write $\delta_{I}^{+}=\delta_{\chi_{I}}^{+}, \delta_{I}^{-}=\delta_{\chi_{I}}^{-}$in short notation. We also put $D_{t}^{+}=\delta_{[0, t)}^{+}, D_{t}^{-}=\delta_{[0, t)}^{-}, t \geq 0$. The operator process $\left\{D_{t}^{+}\right\}_{t \geq 0}$ (resp. $\left.\left\{D_{t}^{-}\right\}_{t \geq 0}\right)$ in $(\mathcal{A}, \phi)$ is called the creation process (resp. the annihilation process).

Let $\left(P_{h}, Q_{h}\right), h \in \mathcal{H}_{1}$ be the canonical pair defined by

$$
\left\{\begin{array}{l}
Q_{h}=\delta_{h}^{+}+\delta_{h}^{-} \\
P_{h}=i\left(\delta_{h}^{+}-\delta_{h}^{-}\right)
\end{array}\right.
$$

Here $i$ is the imaginary unit in $\mathbb{C}$. Let us write $Q_{I}=Q_{\chi_{I}}$ and $P_{I}=P_{\chi_{I}}$ in short notation. Put $Q_{t}=Q_{[0, t)}$ and $P_{t}=P_{[0, t)}$, then we get a noncommuting family $\left\{Q_{t}\right\}_{t \geq 0}$ (resp. $\left\{P_{t}\right\}_{t \geq 0}$ ) of self-adjoint operators. The noncommutativity of the family $\left\{Q_{t}\right\}_{t \geq 0}$ can be checked as follows. For $0<s<t$, we have 


$$
\begin{aligned}
Q_{s} Q_{t} \Omega & =Q_{s} \chi_{[0, t)} \\
& =\chi_{[0, s)} \triangleright \chi_{[0, t)}+\left(\int d t_{1} \overline{\chi_{[0, s)}\left(t_{1}\right)} \chi_{[0, t)}\left(t_{1}\right)\right) \Omega \\
& =\chi_{[0, s)} \triangleright \chi_{[0, t)}+s \Omega .
\end{aligned}
$$

In the same way, we have

$$
Q_{t} Q_{s} \Omega=\chi_{[0, t)} \triangleright \chi_{[0, s)}+s \Omega .
$$

The appearing two 2-particle vectors $u=\chi_{[0, s)} \triangleright \chi_{[0, t)}$ and $v=\chi_{[0, t)} \triangleright \chi_{[0, s)}$ are different:

$$
\begin{aligned}
& u\left(t_{1}>t_{2}\right)= \begin{cases}1 & \text { (if } \left.s>t_{1}>t_{2} \geq 0\right) \\
0 & \text { (otherwise) }\end{cases} \\
& v\left(t_{1}>t_{2}\right)= \begin{cases}1 & \text { (if } \left.t>t_{1}>t_{2} \geq 0 \text { and } s>t_{2}\right) \\
0 & \text { (otherwise) }\end{cases}
\end{aligned}
$$

So we get the noncommutativity $Q_{s} Q_{t} \neq Q_{t} Q_{s}, 0<s<t$. Besides the family $\left\{P_{t}\right\}_{t \geq 0}$ is also mutually noncommuting because $\left\{P_{t}\right\}_{t \geq 0}$ is unitarily equivalent to $\left\{Q_{t}\right\}_{t \geq 0}$ as shown in Sect. 4. We call these two processes $\left\{Q_{t}\right\}_{t \geq 0}$ and $\left\{P_{t}\right\}_{t \geq 0}$ the canonical processes.

The known theories of quantum stochastic calculus (see [HuP, ApH, KuS]), i.e. the bosonic, fermionic and free stochastic calculi suggest that we interpret the canonical process $\left\{Q_{t}\right\}_{t \geq 0}$ (resp. $\left\{P_{t}\right\}_{t \geq 0}$ ) as a kind of Brownian motion. We want to justify this interpretation. In the following sections, we investigate the properties of canonical processes $\left\{Q_{t}\right\}_{t \geq 0}$ and $\left\{P_{t}\right\}_{t \geq 0}$ (or equivalently those of creation and annihilation processes $\left\{D_{t}^{+}\right\}_{t \geq 0}$ and $\left\{D_{t}^{-}\right\}_{t \geq 0}$ ).

\section{Independence in Monotone Fock Space}

In this section, we examine the independence structure in the monotone Fock space.

The general definition of "independence" in quantum probability theory was given by Kümmerer (see [Kum] and [Spe]) as follows. Let $L$ be a totally ordered set. $L$ is interpreted as a set of times.

Definition 3.1. A time indexed family $\left\{\mathcal{C}_{l}\right\}_{l \in L}$ of subalgebras of a unital algebra $\mathcal{C}$ is independent in the sense of Kümmerer with respect to a state $\rho$ over $\mathcal{C}$ if

$$
\rho\left(a_{1} a_{2} \cdots a_{k}\right)=\rho\left(a_{1}\right) \rho\left(a_{2}\right) \cdots \rho\left(a_{k}\right)
$$

whenever $a_{i} \in \mathcal{C}_{l_{i}}, i=1,2, \cdots, k$ with $l_{1}<l_{2}<\cdots<l_{k}, k \geq 1$.

Let $\mathcal{A}(I)=W^{*}\left(1, \delta_{I}^{+}, \delta_{I}^{-}\right)$be the von Neumann algebra generated by the creation and annihilation operators $\delta_{I}^{+}, \delta_{I}^{-}$associated to a finite interval $I$, with identity 1 . Let us write $I_{1}<I_{2}<\cdots<I_{k}$ when $t_{1}<t_{2}<\cdots<t_{k}$ for all $t_{1} \in I_{1}, t_{2} \in I_{2}, \cdots, t_{k} \in I_{k}$. Then we have

Theorem 3.1. The pair process $\left\{\left(D_{t}^{+}, D_{t}^{-}\right)\right\}_{t \geq 0}$ has the property of independence of increments in the Kümmerer independence, with respect to the vacuum state $\phi$ : for any $k$, any mutually disjoint finite intervals $I_{1}, I_{2}, \cdots, I_{k}$ with $I_{1}<I_{2}<\cdots<I_{k}$, and any operators $A_{1} \in \mathcal{A}\left(I_{1}\right), A_{2} \in \mathcal{A}\left(I_{2}\right), \cdots, A_{k} \in \mathcal{A}\left(I_{k}\right)$, we have

$$
\phi\left(A_{1} \cdots A_{k}\right)=\phi\left(A_{1}\right) \cdots \phi\left(A_{k}\right)
$$


Proof. We first prove the factorization property in the special case that $A_{i}$ is a monomial $M_{i}$ of $\delta_{I_{i}}^{ \pm}$. So we assume that $A_{i}$ is given by

$$
A_{i}=M_{i}=\delta_{I_{i}}^{\varepsilon_{i, 1}} \delta_{I_{i}}^{\varepsilon_{i, 2}} \cdots \delta_{I_{i}}^{\varepsilon_{i, N_{i}}}
$$

with some signatures $\varepsilon_{i, 1}, \varepsilon_{i, 2}, \cdots, \varepsilon_{i, N_{i}} \in\{+,-\}$. Here we adopt the convention that $N_{i}=0$ means $M_{i}=1$. We adopt the short notation $M_{i}=\prod \delta_{I_{i}}^{\varepsilon}$ to mean such a monomial. Let $\mathcal{H}_{r}(I), r \geq 1$ be the closed subspaces of $\Phi$ defined by

$$
\mathcal{H}_{r}(I)=\left\{f \in L^{2}\left(\Sigma_{r}\right) \mid f(\sigma)=0, \sigma \in \Sigma_{r} \backslash \Sigma_{r}(I)\right\},
$$

with $\Sigma_{r}(I) \equiv\left\{\sigma=\left(s_{1}>s_{2}>\cdots>s_{r}\right) \in \Sigma_{r} \mid s_{i} \in I, \forall i=1,2, \cdots, r\right\}$. Recall that we made the convention that we omit the phrase " $\lambda$-a.e," and hence $f(\sigma)=0$ precisely means $f(\sigma)=0, \lambda$-a.e. $\sigma$. It is easy to see that the followng holds:

$$
\begin{aligned}
\left(\prod \delta_{I_{k}}^{\varepsilon}\right) \Omega & \in \mathbb{C} \Omega \cup \cup_{r=1}^{\infty} \mathcal{H}_{r}\left(I_{k}\right), \\
\left(\prod \delta_{I_{k-1}}^{\varepsilon}\right)\left(\prod \delta_{I_{k}}^{\varepsilon}\right) \Omega & \in \mathbb{C} \Omega \cup \cup_{r=1}^{\infty} \mathcal{H}_{r}\left(I_{k-1} \cup I_{k}\right), \\
\left(\prod \delta_{I_{2}}^{\varepsilon}\right) \cdots\left(\prod \delta_{I_{k-1}}^{\varepsilon}\right)\left(\prod \delta_{I_{k}}^{\varepsilon}\right) \Omega & \in \mathbb{C} \Omega \cup \cup_{r=1}^{\infty} \mathcal{H}_{r}\left(I_{2} \cup \cdots \cup I_{k-1} \cup I_{k}\right) .
\end{aligned}
$$

Let us prove $\phi\left(M_{1} M_{2} \cdots M_{k}\right)=\phi\left(M_{1}\right) \phi\left(M_{2}\right) \cdots \phi\left(M_{k}\right)$ by mathematical induction on $k$. For $k=1$, it is trivial. Let $k \geq 2$ and put $u=\left(M_{2} M_{3} \cdots M_{k}\right) \Omega$, then only two cases are possible:

$$
\left\{\begin{array}{l}
\text { (a): } u \in \mathcal{H}_{r}\left(I_{2} \cup \cdots \cup I_{k}\right) \text { for some } r \geq 1, \text { and } u \neq 0 . \\
\text { (b): } u \in \mathbb{C} \Omega .
\end{array}\right.
$$

First consider case (a), then we get $\delta_{I_{1}}^{+} u=0$ because of

$$
\left(\delta_{I_{1}}^{+} u\right)(s>\sigma)=\left(\chi_{I_{1}} \triangleright u\right)(s>\sigma)=\chi_{I_{1}}(s) u(\sigma)=0 \quad(s>\sigma),
$$

where the last equality comes from $I_{1}<I_{2} \cup \cdots \cup I_{k}$. We also get $\delta_{I_{1}}^{-} u=0$ because of

$$
\left(\delta_{I_{1}}^{-} u\right)(\tau)=\int d s \overline{\chi_{I_{1}}(s)} u(s>\tau)=0,
$$

where the last equality comes from $I_{1} \cap\left(I_{2} \cup \cdots \cup I_{k}\right)=\emptyset$. So when $M_{1}=\prod \delta_{I_{1}}^{\varepsilon} \neq \mathbf{1}$, we have

$$
<\Omega\left|M_{1} M_{2} \cdots M_{k} \Omega\right\rangle=\langle\Omega|\left(\delta_{I_{1}}^{\varepsilon_{1,1}} \delta_{I_{1}}^{\varepsilon_{1,2}} \cdots \delta_{I_{1}}^{\varepsilon_{1, N_{1}-1}}\right) \delta_{I_{1}}^{\varepsilon_{1, N_{1}}} u>=0
$$

because of $\delta_{I_{1}}^{ \pm} u=0$. On the other hand we have

$$
\begin{aligned}
<\Omega \mid M_{1} \Omega & >\left(<\Omega\left|M_{2} \Omega>\cdots<\Omega\right| M_{k} \Omega>\right) \\
= & <\Omega\left|M_{1} \Omega><\Omega\right| M_{2} \cdots M_{k} \Omega> \\
= & <\Omega\left|M_{1} \Omega><\Omega\right| u>=0
\end{aligned}
$$

because (a) implies $\langle\Omega \mid u\rangle=0$. Therefore case (a) with $M_{1}=\prod \delta_{I_{1}}^{\varepsilon} \neq 1$ implies the factorization

$$
\phi\left(M_{1} M_{2} \cdots M_{k}\right)=\phi\left(M_{1}\right) \phi\left(M_{2}\right) \cdots \phi\left(M_{k}\right) .
$$


Besides case (a) with $M_{1}=\prod \delta_{I_{1}}^{\varepsilon}=1$ also implies the factorization.

In case (b), the vector $u$ must be of the form $u=a \Omega$ with some scalar $a \in \mathbb{C}$, and hence we get

$$
\begin{aligned}
<\Omega \mid M_{1} M_{2} & \cdots M_{k} \Omega>=<\Omega \mid M_{1} a \Omega> \\
= & <\Omega\left|M_{1} \Omega>a=<\Omega\right| M_{1} \Omega><\Omega \mid a \Omega> \\
& =<\Omega\left|M_{1} \Omega><\Omega\right| M_{2} \cdots M_{k} \Omega> \\
& =<\Omega\left|M_{1} \Omega><\Omega\right| M_{2} \Omega>\cdots<\Omega \mid M_{k} \Omega>.
\end{aligned}
$$

So in both cases (a) and (b), we have the factorization property. By the induction on $k$, we conclude that the factorization property holds for all $k \geq 1$ and all monomials $M_{1}=\prod \delta_{I_{1}}^{\varepsilon}, M_{2}=\prod \delta_{I_{2}}^{\varepsilon}, \cdots, M_{k}=\prod \delta_{I_{k}}^{\varepsilon}$.

Next we must show that the factorization property holds for all polynomials $P_{i}$ of the creation and annihilation operators $\delta_{I_{i}}^{ \pm}$. That is, $A_{i}$ is supposed to be of the form

$$
A_{i}=P_{i}=\sum_{l_{i}=1}^{L_{i}} a_{l_{i}}^{(i)} M_{l_{i}}^{(i)}
$$

with some monomials $M_{l_{i}}^{(i)}$ of $\delta_{I_{i}}^{ \pm}$and some complex scalars $a_{l_{i}}^{(i)} \in \mathbb{C}$. In this case, the factorization property is easily obtained from the multi-linearity of the map $\left(P_{1}, P_{2}, \cdots, P_{k}\right) \mapsto \phi\left(P_{1} P_{2} \cdots P_{k}\right)$.

Finally let us show the factorization property for arbitrary $A_{1} \in \mathcal{A}\left(I_{1}\right), \cdots, A_{k} \in$ $\mathcal{A}\left(I_{k}\right)$. Since $A_{1}$ is the limit, in the weak operator topology, of some polynomials $\left\{P_{1}^{(n)}\right\}_{n \in \mathbb{N}}$ of $\delta_{I_{1}}^{ \pm}$, we get

$$
\begin{aligned}
\phi\left(A_{1} P_{2} \cdots P_{k}\right) & =\lim _{n \rightarrow \infty} \phi\left(P_{1}^{(n)} P_{2} \cdots P_{k}\right) \\
& =\lim _{n \rightarrow \infty} \phi\left(P_{1}^{(n)}\right) \phi\left(P_{2}\right) \cdots \phi\left(P_{k}\right)=\phi\left(A_{1}\right) \phi\left(P_{2}\right) \cdots \phi\left(P_{k}\right) .
\end{aligned}
$$

Using the convergence

$$
A_{1} \cdots A_{i-1} P_{i}^{(n)} P_{i+1} \cdots P_{k} \rightarrow A_{1} \cdots A_{i-1} A_{i} P_{i+1} \cdots P_{k}
$$

in the weak operator topology and repeating the preceding discussion, we get

$$
\phi\left(A_{1} A_{2} \cdots A_{k}\right)=\phi\left(A_{1}\right) \phi\left(A_{2}\right) \cdots \phi\left(A_{k}\right) .
$$

This completes the proof.

As a corollary of Theorem 3.1 , we get

Corollary 3.1. The canonical process $\left\{Q_{t}\right\}_{t \geq 0}$ (resp. $\left.\left\{P_{t}\right\}_{t \geq 0}\right)$ is a process with independent increments in the Kümmerer independence, with respect to the vacuum state $\phi$. 


\section{Probability Distribution of Canonical Process}

In this section, we calculate the probability distribution associated to the canonical process $\left\{Q_{t}\right\}_{t \geq 0}$ (and that to $\left\{P_{t}\right\}_{t \geq 0}$ ).

Let us calculate the probability distribution $\mu_{I}$ of an increment $Q_{I}=Q_{b}-Q_{a}$, $I=[a, b), 0 \leq a<b$, of the canonical process $\left\{Q_{t}\right\}_{t \geq 0}$ under the vacuum state $\phi$. We shall calculate the probability distribution $\mu_{I}$ by the moment method. Let us calculate the $p^{\text {th }}$ moment $m_{p}$ of $Q_{I}$ in the vacuum state $\phi$. We have

$$
\begin{aligned}
m_{p}=\phi\left(Q_{I}^{p}\right) & =\phi\left(\left(\delta_{I}^{+}+\delta_{I}^{-}\right)^{p}\right) \\
& =\sum_{\left(\varepsilon_{1}, \varepsilon_{2}, \cdots, \varepsilon_{p}\right) \in\{+,-\}^{p}} \phi\left(\delta_{I}^{\varepsilon_{1}} \delta_{I}^{\varepsilon_{2}} \cdots \delta_{I}^{\varepsilon_{p}}\right) .
\end{aligned}
$$

Note that since $\delta_{I}^{-} \Omega=0$, the set $\mathbb{C} \Omega \cup \bigcup_{r=1}^{\infty} \mathcal{H}_{r}$ is stable under the action of $\delta_{I}^{+}$and $\delta_{I}^{-}$. Hence the term

$$
\phi\left(\delta_{I}^{\varepsilon_{1}} \delta_{I}^{\varepsilon_{2}} \cdots \delta_{I}^{\varepsilon_{p}}\right)=<\Omega \mid \delta_{I}^{\varepsilon_{1}} \delta_{I}^{\varepsilon_{2}} \cdots \delta_{I}^{\varepsilon_{p}} \Omega>
$$

survives only when $\delta_{I}^{\varepsilon_{1}} \delta_{I}^{\varepsilon_{2}} \cdots \delta_{I}^{\varepsilon_{p}} \Omega \in \mathbb{C} \Omega$. Such a requirement imposes some constraint on a sequence $\varepsilon=\left(\varepsilon_{1}, \varepsilon_{2}, \cdots, \varepsilon_{p}\right)$ of signatures,+- . It is easy to see that $m_{p}=0$ for odd $p$. Let us calculate the $p^{\text {th }}$ moment $\phi\left(Q_{I}^{p}\right)$ for even $p=2 k$. At first we see that, for

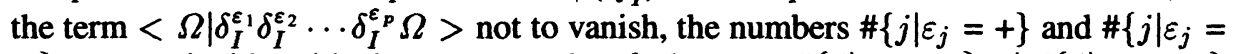
$-\}$ must coincide with the same number $k$, because $\#\left\{j \mid \varepsilon_{j}=+\right\} \neq \#\left\{j \mid \varepsilon_{j}=-\right\}$ implies either $\delta_{I}^{\varepsilon_{1}} \delta_{I}^{\varepsilon_{2}} \cdots \delta_{I}^{\varepsilon_{p}} \Omega=0$ or $0 \neq \delta_{I}^{\varepsilon_{1}} \delta_{I}^{\varepsilon_{2}} \cdots \delta_{I}^{\varepsilon_{p}} \Omega \in \bigcup_{r=1}^{\infty} \mathcal{H}_{r}$. Furthermore more constraints must be imposed on a sequence $\varepsilon=\left(\varepsilon_{1}, \varepsilon_{2}, \cdots, \varepsilon_{p}\right)$ as follows. For the term $<\Omega \mid \delta_{I}^{\varepsilon_{1}} \delta_{I}^{\varepsilon_{2}} \cdots \delta_{I}^{\varepsilon_{p}} \Omega>$ not to vanish, the vector $\delta_{I}^{\varepsilon_{1}} \delta_{I}^{\varepsilon_{l+1}} \cdots \delta_{I}^{\varepsilon_{p}} \Omega$ must not vanish for each $l=1,2, \cdots, p$. So the number $\#\left\{j \mid j \geq l, \varepsilon_{j}=-\right\}$ of "times of annihilation" must not exceed the number $\#\left\{j \mid j \geq l, \varepsilon_{j}=+\right\}$ of "times of creation," for each $l=1,2, \cdots, p$. Therefore, for the purpose of calculation of the $p^{\text {th }}$ moment $\phi\left(Q_{I}^{p}\right)$ with $p=2 k$, we have only to take into account the sequences $\varepsilon=\left(\varepsilon_{1}, \varepsilon_{2}, \cdots, \varepsilon_{p}\right)$ satisfying

$$
\begin{aligned}
& \text { (a) } \#\left\{j \mid \varepsilon_{j}=+\right\}=\#\left\{j \mid \varepsilon_{j}=-\right\}=k, \\
& \text { (b) } \#\left\{j \mid j \geq l, \varepsilon_{j}=+\right\}-\#\left\{j \mid j \geq l, \varepsilon_{j}=-\right\} \geq 0, \quad \forall l=1,2, \cdots, p .
\end{aligned}
$$

A sequence $\varepsilon$ satisfying these conditions is called an admissible sequence. Such a sequence can be visualized in more pictorial language. We connect $i$ and $j, i<j$, by a line if and only if

$$
\begin{aligned}
& \text { (i) } \#\left\{h \mid l \leq h \leq j, \varepsilon_{h}=+\right\}-\#\left\{h \mid l \leq h \leq j, \varepsilon_{h}=-\right\}>0, \quad i<\forall l \leq j \text {, } \\
& \text { (ii) } \#\left\{h \mid i \leq h \leq j, \varepsilon_{h}=+\right\}-\#\left\{h \mid i \leq h \leq j, \varepsilon_{h}=-\right\}=0 .
\end{aligned}
$$

For example $\varepsilon=(-,-,-,+,-,+,+,+,-,+,-,-,+,+)$ is visualized as in Fig. 1. Such

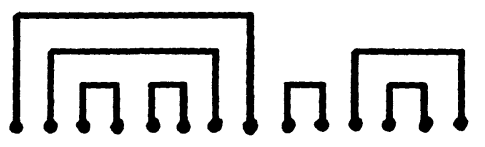

Fig. 1. An admissible figure $g$

a figure $g=g(\varepsilon)$ corresponding to an admissible sequence $\varepsilon=\left(\varepsilon_{1}, \varepsilon_{2}, \cdots, \varepsilon_{p}\right)$ is called an admissible figure. There is the bijective correspondence between the admissible 
sequences $\{\varepsilon \mid$ admissible $\}$ and the admissible figures $\{g \mid$ admissible $\}$. Denote by $G(p)$ the set of all admissible figures corresponding to the admissible sequences of even length $p=2 k$. Put $G=\cup_{k=1}^{\infty} G(2 k)$. For each finite time interval $I=[a, b)$ and each admissible figure $g \in G(2 k)$ associated with the sequence $\left(\varepsilon_{1}, \varepsilon_{2}, \cdots, \varepsilon_{2 k}\right)$ of signatures, put $\delta_{I}^{g} \equiv \delta_{I}^{\varepsilon_{1}} \delta_{I}^{\varepsilon_{2}} \cdots \delta_{I}^{\varepsilon_{2 k}}$. Then the $2 k^{\text {th }}$ moment $m_{2 k}$ is given by the formula

$$
m_{2 k}=\sum_{g \in G(2 k)}<\Omega \mid \delta_{I}^{g} \Omega>.
$$

Denote by $\langle g ; I\rangle$ the vacuum expectation $\langle\Omega| \delta_{I}^{g} \Omega>$ of $\delta_{I}^{g}$. Define the function $d(g)$ by $d(g)=k$ for $g=g(\varepsilon)$ with $\varepsilon=\left(\varepsilon_{1}, \varepsilon_{2}, \cdots, \varepsilon_{2 k}\right)$. Then we can prove the following.

Theorem 4.1. $\langle g ; I>$ is the monomial, with order $d(g)$, of the length $\lambda(I)$ of time interval I, i.e.

$$
\langle g ; I\rangle=a(g) \lambda(I)^{d(g)} .
$$

Here the system of coefficients $\{a(g)\}_{g \in G}$ is determined by the recurrence relation:

$$
\begin{array}{ll}
\text { (1) } a(g)=1 & \text { (if } g=\square) ; \\
\text { (2) } a(g)=a\left(g_{1}\right) a\left(g_{2}\right) \cdots a\left(g_{l}\right) & \text { (if } \left.g=g_{1} g_{2} \cdots g_{l}\right) ; \\
\text { (3) } a(g)=\frac{1}{d\left(g_{1}\right)+1} a\left(g_{1}\right) & \left(\text { if } g=\sqrt{g_{1}}\right) .
\end{array}
$$

Before proving Theorem 4.1, we prepare two lemmas.

Lemma 4.1. $\delta_{I}^{g} \Omega=<g ; I>\Omega$.

Proof. Note that $\delta_{I}^{g} \Omega \in \mathbb{C} \Omega$ because $g$ is an admissible figure. Since we have $<$ $\Omega \mid \delta_{I}^{g} \Omega>=<g ; I>$ by the definition of $\left\langle g ; I>\right.$, we conclude $\delta_{I}^{g} \Omega=<g ; I>\Omega$.

We adopt the short notation $\{\geq s\}$ to mean the set $\left\{t \in \mathbb{R}_{+} \mid t \geq s\right\}$.

Lemma 4.2. For each $r$-particle vector $u=u(\sigma)=u\left(s_{1}>s_{2}>\cdots>s_{r}\right)$ with $r \geq 1$, we have $\left(\delta_{I}^{g} u\right)(\sigma)=<g ; I \cap\left\{\geq s_{1}\right\}>u(\sigma)$.

Proof. Let us prove the equality by the mathematical induction on $d(g)$. First consider the case of $d(g)=1$, i.e. $g=\square$. Since we have

$$
\begin{aligned}
\left(\delta_{I}^{-} \delta_{I}^{+} u\right)(\sigma) & =\int d t \overline{\chi_{I}(t)}\left(\chi_{I} \triangleright u\right)(t>\sigma) \\
& =\int_{t>s_{1}} d t \overline{\chi_{I}(t)} \chi_{I}(t) u(\sigma)=\lambda\left(I \cap\left\{\geq s_{1}\right\}\right) u(\sigma)
\end{aligned}
$$

and

$$
\begin{aligned}
<g ; I \cap\left\{\geq s_{1}\right\}>u(\sigma) & =<\Omega \mid \delta_{I \cap\left\{\geq s_{1}\right\}}^{-} \delta_{I \cap\left\{\geq s_{1}\right\}}^{+} \Omega>u(\sigma) \\
& =\lambda\left(I \cap\left\{\geq s_{1}\right\}\right) u(\sigma),
\end{aligned}
$$

we get the desired equality.

Now let us make the inductive hypothesis that the equality $\left(\delta_{I}^{g} u\right)(\sigma)=$ $<g ; I \cap\left\{\geq s_{1}\right\}>u(\sigma)$ holds for all $g$ such that $d(g)<k$. Let us show the equality for $d(g)=k$. Consider the case of $g=g_{1} g_{2} \cdots g_{l}$ with $l \geq 2$. Put 


$$
\varphi_{g_{1} \cdots g_{l-1}}(\sigma)=<g_{1} \cdots g_{l-1} ; I \cap\left\{\geq s_{1}\right\}>\quad \text { and } \quad \varphi_{g_{l}}(\sigma)=<g_{l} ; I \cap\left\{\geq s_{1}\right\}>
$$

with $\sigma=\left(s_{1}>s_{2}>\cdots>s_{r}\right)$. Since $d\left(g_{l}\right)<k$, we have

$$
\left(\delta_{I}^{g_{l}} u\right)(\sigma)=\varphi_{g_{l}}(\sigma) u(\sigma)
$$

by the inductive hypothesis. Furthermore, since $d\left(g_{1} \cdots g_{l-1}\right)<k$, we have

$$
\left(\delta_{I}^{g_{1} \cdots g_{l-1}} v\right)(\sigma)=\varphi_{g_{1} \cdots g_{l-1}}(\sigma) v(\sigma) \text { for } \quad v=\varphi_{g_{l}} u
$$

by the inductive hypothesis. Hence we get

$$
\begin{aligned}
\left(\delta_{I}^{g} u\right)(\sigma) & =\left(\delta_{I}^{g_{1} \cdots g_{l-1}} \delta_{I}^{g_{l}} u\right)(\sigma)=\left(\delta_{I}^{g_{1} \cdots g_{l-1}} \varphi_{g_{l}} u\right)(\sigma) \\
& =\left(\delta_{I}^{g_{1} \cdots g_{l-1}} v\right)(\sigma)=\varphi_{g_{1} \cdots g_{l-1}}(\sigma) v(\sigma) \\
& =<g_{1} \cdots g_{l-1} ; I \cap\left\{\geq s_{1}\right\}><g_{l} ; I \cap\left\{\geq s_{1}\right\}>u(\sigma)
\end{aligned}
$$

On the other hand, we have

$$
\begin{aligned}
<g ; I \cap\{ & \left.\geq s_{1}\right\}>u(\sigma)=<\Omega \mid \delta_{I \cap\left\{\geq s_{1}\right\}}^{g_{1} \cdots g_{g_{1}-1}} \delta_{I \cap\left\{\geq s_{1}\right\}}^{g_{l}} \Omega>u(\sigma) \\
& =<\Omega \mid \delta_{I \cap\left\{\geq s_{1}\right\}}^{g_{1} \cdots g_{l-1}}<g_{l} ; I \cap\left\{\geq s_{1}\right\}>\Omega>u(\sigma) \\
& =<g_{1} \cdots g_{l-1} ; I \cap\left\{\geq s_{1}\right\}><g_{l} ; I \cap\left\{\geq s_{1}\right\}>u(\sigma)
\end{aligned}
$$

where the second equality comes from Lemma 4.1. Combining these, we get the desired equality.

Finally let us consider the case of $g=\sqrt{g_{1}}$. Put $\varphi_{g_{1}}(t>\sigma)=<g_{1} ; I \cap\{\geq t\}>$ and $\psi_{g_{1}}(t)=<g_{1} ; I \cap\left\{\geq s_{1}\right\} \cap\{\geq t\}>$. Since $d\left(g_{1}\right)<k$, we have

$$
\left(\delta_{I}^{g_{1}} v\right)(t>\sigma)=\varphi_{g_{1}}(t>\sigma) v(t>\sigma) \text { for } \quad v=\chi_{I} \triangleright u
$$

by the inductive hypothesis. By the same reason, we have

$$
\left(\delta_{I \cap\left\{\geq s_{1}\right\}}^{g_{1}} \chi_{I \cap\left\{\geq s_{1}\right\}}\right)(t)=\psi_{g_{1}}(t) \chi_{I \cap\left\{\geq s_{1}\right\}}(t) .
$$

Hence we have

$$
\begin{aligned}
<g ; I \cap\left\{\geq s_{1}\right\}> & =\langle\Omega| \delta_{I \cap\left\{\geq s_{1}\right\}}^{-} \delta_{I \cap\left\{\geq s_{1}\right\}}^{g_{1}} \delta_{I \cap\left\{\geq s_{1}\right\}}^{+} \Omega> \\
& =\langle\Omega| \delta_{I \cap\left\{\geq s_{1}\right\}}^{-} \delta_{I \cap\left\{\geq s_{1}\right\}}^{g_{1}} \chi_{I \cap\left\{\geq s_{1}\right\}}> \\
& =\langle\Omega| \delta_{I \cap\left\{\geq s_{1}\right\}}^{-} \psi_{g_{1}} \chi_{I \cap\left\{\geq s_{1}\right\}}> \\
& =\int d t \overline{\chi_{I \cap\left\{\geq s_{1}\right\}}} \psi_{g_{1}}(t) \chi_{I \cap\left\{\geq s_{1}\right\}}(t) \\
& =\int_{I \cap\left\{\geq s_{1}\right\}} d t<g_{1} ; I \cap\{\geq t\}>.
\end{aligned}
$$

On the other hand 


$$
\begin{aligned}
\left(\delta_{I}^{g} u\right)(\sigma) & =\left(\delta_{I}^{-} \delta_{I}^{g_{1}} \delta_{I}^{+} u\right)(\sigma)=\left(\delta_{I}^{-} \delta_{I}^{g_{1}}\left(\chi_{I} \triangleright u\right)\right)(\sigma) \\
& =\left(\delta_{I}^{-} \delta_{I}^{g_{1}} v\right)(\sigma)=\left(\delta_{I}^{-} \varphi_{g_{1}} v\right)(\sigma) \\
& =\int d t \overline{\chi_{I}(t)} \varphi_{g_{1}}(t>\sigma) v(t>\sigma) \\
& =\int_{t>s_{1}} d t \overline{\chi_{I}(t)}<g_{1} ; I \cap\{\geq t\}>\chi_{I}(t) u(\sigma) \\
& =\int_{I \cap\left\{\geq s_{1}\right\}} d t<g_{1} ; I \cap\{\geq t\}>u(\sigma) .
\end{aligned}
$$

Combining these, we get the desired equality. Since we have checked the equality in each cases, this completes the proof.

Proof of Theorem 4.1. Let us prove the statement by the mathematical induction on $d(g)$. For the case of $d(g)=1$, i.e. $g=\square$, we have

$$
\langle\downarrow ; I\rangle=\left\langle\Omega \mid \delta_{I}^{-} \delta_{I}^{+} \Omega\right\rangle=\int d \overline{\chi_{I}(t)} \chi_{I}(t)=\lambda(I),
$$

and hence $\langle\downarrow ; I\rangle=\lambda(I)$ and $a(\coprod)=1$. In the case of $g=g_{1} g_{2} \cdots g_{l}$, we have

$$
\begin{aligned}
<g_{1} \cdots g_{l} ; I> & =\langle\Omega| \delta_{I}^{g_{1} \cdots g_{l-1}} \delta_{I}^{g_{l}} \Omega>=\left\langle\Omega \mid \delta_{I}^{g_{1} \cdots g_{l-1}}<g_{l} ; I>\Omega\right\rangle \\
& =\langle\Omega| \delta_{I}^{g_{1} \cdots g_{l-1}} \Omega><g_{l} ; I> \\
& =a\left(g_{1} \cdots g_{l-1}\right) \lambda(I)^{d\left(g_{1} \cdots g_{l-1}\right)} a\left(g_{l}\right) \lambda(I)^{d\left(g_{l}\right)} \\
& =a\left(g_{1}\right) \cdots a\left(g_{l-1}\right) a\left(g_{l}\right) \lambda(I)^{d\left(g_{1} \cdots g_{l}\right)},
\end{aligned}
$$

where the second equality comes from Lemma 4.1. Hence we get

$$
\left\langle g_{1} \cdots g_{l} ; I\right\rangle=a\left(g_{1} \cdots g_{l}\right) \lambda(I)^{d\left(g_{1} \cdots g_{l}\right)}
$$

and $a\left(g_{1} \cdots g_{l}\right)=a\left(g_{1}\right) \cdots a\left(g_{l}\right)$. In the case of $g=\sqrt{g_{1}}$, we have

$$
\begin{aligned}
\left\langle g_{1} ; I\right\rangle & =\left\langle\Omega \mid \delta_{I}^{-} \delta_{I}^{g_{1}} \delta_{I}^{+} \Omega\right\rangle \\
& =\int d s \overline{\chi_{I}(s)}\left(\delta_{I}^{g_{1}} \chi_{I}\right)(s)=\int d s \overline{\chi_{I}(s)}<g_{1} ; I \cap\{\geq s\}>\chi_{I}(s) \\
& =\int_{I} d s\left\langle g_{1} ; I \cap\{\geq s\}\right\rangle=\int_{I} d s a\left(g_{1}\right)(b-s)^{d\left(g_{1}\right)} \\
& =a\left(g_{1}\right)\left[-\frac{(b-s)^{d\left(g_{1}\right)+1}}{d\left(g_{1}\right)+1}\right]_{a}^{b}=\left(\frac{a\left(g_{1}\right)}{d\left(g_{1}\right)+1}\right)(b-a)^{d\left(g_{1}\right)+1} .
\end{aligned}
$$

Here the third equality comes from Lemma 4.2 and $I$ is assumed to be of the form $I=[a, b)$. Hence we get $\left\langle\sqrt{g_{1}} ; ;\right\rangle=a\left(g_{1}\right)(b-a)^{d\left(g_{1}\right)+1}$ and $a\left(\sqrt{g_{1}}\right)=\frac{a\left(g_{1}\right)}{d\left(g_{1}\right)+1}$. By the induction on $d(g)$, we complete the proof.

From Theorem 4.1, we get the expression for the moment $m_{2 k}$ :

$$
m_{2 k}=\left(\sum_{g \in G(2 k)} a(g)\right) \lambda(I)^{k} .
$$


Put $a_{2 k}=\sum_{g \in G(2 k)} a(g)$ and $a_{0}=1$, then the sequence $\left\{a_{2 k}\right\}_{k \in \mathbb{N}}$ satisfies the following recurrence equation:

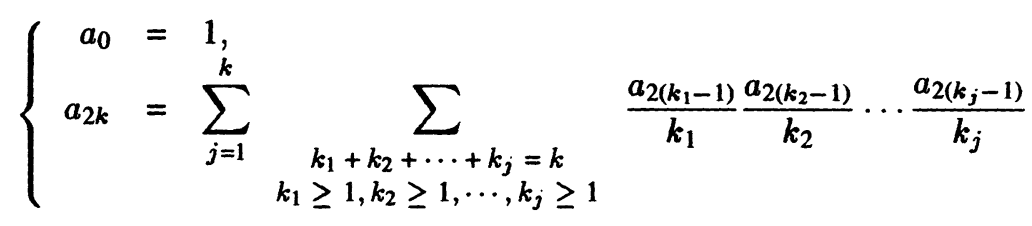

because of the recurrence relation for $\{a(g)\}_{g \in G}$ in Theorem 4.1. Such a form of recurrence equation was already obtained and solved in a previous paper [Mur1].

Following [Mur1], we briefly sketch the process solving the above recurrence equation. At first let us solve it with a formal calculation. Let $f(s)=\sum_{k=0}^{\infty} a_{2 k} s^{k}$ be the (formal) generating fuction for the sequence $\left\{a_{2 k}\right\}_{k \in \mathrm{N}}$. Let $g(s)=\int_{0}^{s} f(s) d s$, then we have

$$
\begin{aligned}
& f(s)=1+\sum_{k=1}^{\infty}\left(\sum_{j=1}^{k} \sum_{\substack{k_{1}+k_{2}+\cdots+k_{j}=k \\
k_{1} \geq 1, k_{2} \geq 1, \cdots, k_{j} \geq 1}} \frac{a_{2\left(k_{1}-1\right)}}{k_{1}} \frac{a_{2\left(k_{2}-1\right)}}{k_{2}} \ldots \frac{a_{2\left(k_{j}-1\right)}}{k_{j}}\right) s^{k} \\
& =1+\sum_{j=1}^{\infty}\left(\sum_{\substack { k=j \\
\begin{subarray}{c}{k_{1}+k_{2}+\cdots+k_{j}=k \\
k_{1} \geq 1, k_{2} \geq 1, \cdots, k_{j} \geq 1{ k = j \\
\begin{subarray} { c } { k _ { 1 } + k _ { 2 } + \cdots + k _ { j } = k \\
k _ { 1 } \geq 1 , k _ { 2 } \geq 1 , \cdots , k _ { j } \geq 1 } }\end{subarray}}^{\infty} \frac{a_{2\left(k_{1}-1\right)}}{k_{1}} s^{k_{1}} \frac{a_{2\left(k_{2}-1\right)}}{k_{2}} s^{k_{2}} \ldots \frac{a_{2\left(k_{j}-1\right)}}{k_{j}} s^{k_{j}}\right) \\
& =1+\sum_{j=1}^{\infty} g(s)^{j} \\
& =\frac{1}{1-g(s)} \text {. }
\end{aligned}
$$

Hence we get the differential equation for $g$ :

$$
g^{\prime}(s)=\frac{1}{1-g(s)}, \quad g(0)=0 .
$$

Differentiating this equation and eliminating $g(s)$, we get the differential equation for $f:$

$$
f^{\prime}(s)=f(s)^{3}, \quad f(0)=1
$$

and the solution $f(s)=\frac{1}{\sqrt{1-2 s}}$. Expanding $f(s)$ to the binomial series, we finally get

$$
a_{2 k}=\left(\begin{array}{c}
-\frac{1}{2} \\
k
\end{array}\right)(-2)^{k} .
$$

Though we obtained the expression for $a_{2 k}$ by a formal calculation, we can a posteriori check that $a_{2 k}=\left(\begin{array}{c}-\frac{1}{2} \\ k\end{array}\right)(-2)^{k}$ is certainly the unique solution of the recurrence equation. The sequence $\left\{a_{2 k}\right\}_{k \in \mathbb{N}}$ uniquely determines a probability measure $\mu$ on the real line $\mathbb{R}$ : 


$$
\frac{d \mu}{d \lambda}(x)= \begin{cases}\frac{1}{\pi \sqrt{(\sqrt{2}+x)(\sqrt{2}-x)}}(-\sqrt{2}<x<\sqrt{2}) \\ 0 \quad \text { (otherwise) }\end{cases}
$$

as the unique solution of Hamburger's moment problem. This can be checked with a direct calculation of the moment generating function associated to the above probability measure $\mu$ [Mur1]. We call the above probability measure $\mu$ the standard arcsine law because it is the normalization of the usual arcsine law $\mu_{0}$ (see [Fel]),

$$
\frac{d \mu_{0}}{d \lambda}(x)= \begin{cases}\frac{1}{\pi \sqrt{x(1-x)}}(0<x<1), \\ 0 \quad \text { (otherwise) }\end{cases}
$$

into a probability distribution with mean 0 and variance 1 . Since $m_{2 k}=a_{2 k} \lambda(I)^{k}$, the sequence $\left\{m_{2 k}\right\}_{k \in \mathbb{N}}$ uniquely determines a probability distribution $\mu_{I}$ which is given by $\mu_{I}(d x)=\mu(d x / \sqrt{\lambda(I)})$.

Now we finally get the probability distribution $\mu_{I}$ of an increment of the canonical process $\left\{Q_{t}\right\}_{t \geq 0}$.

Theorem 4.2. The probability distribution $\mu_{I}$ of an increment $Q_{I}=Q_{t}-Q_{s}, I=[s, t)$, $0 \leq s<t$, in the vacuum state $\phi$ is the arcsine law normalized to mean 0 and variance $t-s$ :

$$
\frac{d \mu_{I}}{d \lambda}(x)= \begin{cases}\frac{1}{\pi \sqrt{(\sqrt{2(t-s)}+x)(\sqrt{2(t-s)}-x)}}(-\sqrt{2(t-s)}<x<\sqrt{2(t-s)}) \\ 0 \quad \text { (otherwise). }\end{cases}
$$

For another canonical process $\left\{P_{t}\right\}_{t \geq 0}$, we get the same result as follows. Let us define a unitary operator $\mathcal{F}$ on $\Phi$ by $\mathcal{F} u=i^{r} u, u \in \mathcal{H}_{r}, r=0,1,2, \cdots$, where $i$ is the imaginary unit. It is an analogue of the Fourier-Wiener transform in the theory of classical Brownian motion. It is easy to see that $\mathcal{F} Q_{I} \mathcal{F}^{-1}=P_{I}, \mathcal{F} P_{I} \mathcal{F}^{-1}=-Q_{I}$ and $\mathcal{F} \Omega=\Omega$. So it gives rise to the unitary equivalence between the triples $\left(\Phi, Q_{I}, \Omega\right)$ and $\left(\Phi, P_{I}, \Omega\right)$. Hence Theorem 4.2 holds in the form that $\left\{Q_{t}\right\}_{t>0}$ is replaced with $\left\{P_{t}\right\}_{t \geq 0}$.

Corollary 4.1. The probability distribution of an increment $P_{I}=P_{t}-P_{s}, I=[s, t)$, $0 \leq s<t$, in the vacuum state $\phi$ is the arcsine law normalized to mean 0 and variance $t-s$.

We remark that the standard arcsine law already appeared as the limit distribution of a noncommutative de Moivre-Laplace theorem [Mur1]. This noncommutative de Moivre-Laplace theorem was constructed on the "discrete time" monotone Fock space with noncommutative random walks $\left\{q_{n}\right\}_{n \in \mathbb{N}}$ and $\left\{p_{n}\right\}_{n \in \mathbb{N}}$ (see [Mur1] for details). The canonical process $\left\{Q_{t}\right\}_{t \geq 0}$ (resp. $\left\{P_{t}\right\}_{t \geq 0}$ ) can be viewed as a scaling limit of random walk $\left\{q_{n}\right\}_{n \in \mathbb{N}}$ (resp. $\left\{p_{n}\right\}_{n \in \mathbb{N}}$ ). From this point of view and the independence result in the previous section, we conclude that $\left\{Q_{t}\right\}_{t \geq 0}$ (resp. $\left\{P_{t}\right\}_{t \geq 0}$ ) can be viewed as a noncommutative analogue of Brownian motion.

In Fig. 2, we plot the graph of probability distribution $\mu_{t}=\mu_{[0, t)}$ of the canonical process $Q_{t}, t \geq 0$. The distributions at time $t=1 / 4,1,4$ are sampled.

We are now trying to develop a quantum stochastic calculus on the monotone Fock space which will be presented in an upcoming paper [Mur2]. 


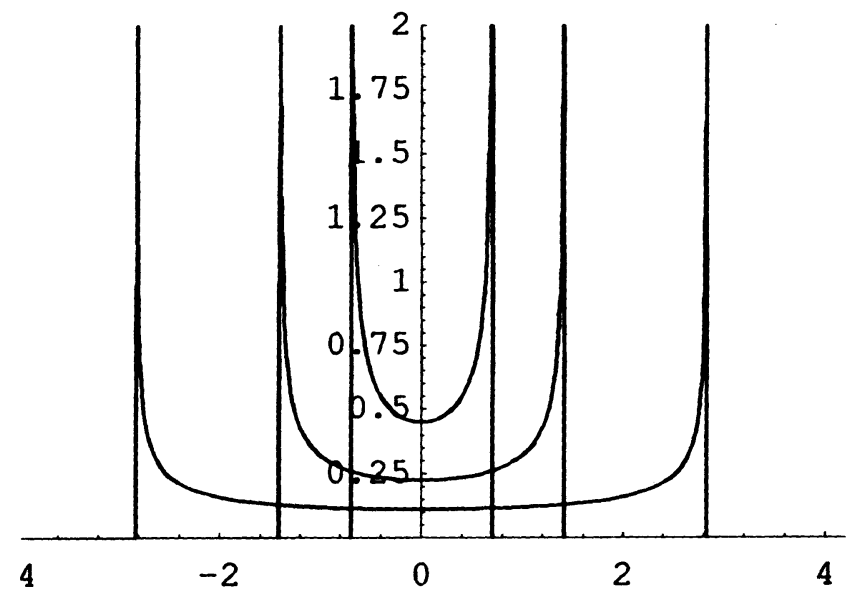

Fig. 2. The distribution of noncommutative Brownian motion $\left\{Q_{t}\right\}_{t \geq 0}$

\section{References}

[ApH] Applebaum, D. B., Hudson, R. L.: Fermion Ito's formula and stochastic evolution. Commun. Math. Phys. 96, 473-496 (1986)

[BSW] Barnett, C., Streater, R. F., Wilde, I. F.: The Ito-Clifford integral. J. Funct. Anal. 48, 172-212 (1982)

[BoS] Bozéjko,M., Speicher, R.: An example of a generalized Brownian motion. Commun. Math. Phys. 137, 519-531 (1991)

[CuH] Cushen, D. D., Hudson, R. L.: A quantum mechanicalcentral limit theorem. J. Appl. Prob. 8, 454-469 (1971)

[Fel] Feller, W.: An Introduction to Probability Theory and Its Applications, Volume 1. New York: John Wiley \& Sons, 1968

[GvW] Giri, N., von Waldenfels, W.: An algebraic version of the central limit theorem. Z. Wahr. Verw. Gebiete 42, 129-134(1978)

[Hud] Hudson, R. L.: A quantum mechanical central limit theorem for anticommuting observables. J. Appl. Prob. 10, 502-509(1973)

[HuP] Hudson, R. L., Parthasarathy, K. R.: Quantum Ito's formula and stochastic evolution. Commun. Math. Phys. 93, 301-323(1984)

[Kum] Kümmerer, B.: Markov dilations and non-commutative Poisson processes. Preprint

[KuS] Kümmerer, B., Speicher, R.: Stochastic integration on the Cuntz-algebra $\mathcal{O}_{\infty}$. J. Funct. Anal. 103, 372-408 (1992)

[Maa] Maassen, H.: Quantum Markov processes on Fock space described by integral kernels. In: Quantum Probability and Applications II. Proceedings, Heidelberg 1984, ed. by L. Accardi and W. von Waldenfels. Lecture Notes in Mathematics 1136, Berlin - Heidelberg - New York: Springer, 1985, pp.361-374

[Mey] Meyer, P. A.: Quantum Probability for Probabilists. Lecture Notes in Mathematics 1538, Berlin Heidelberg - New York: Springer, 1993

[Mur1] Muraki, N.: A new example of noncommutative "de Moivre-Laplace theorem." In: Probability Theory and Mathematical Statistics. Proceedings of the Seventh Japan- Russia Symposium, Tokyo 1995, ed. by S. Watanabe, M. Fukushima, Yu. V. Prohorov and A. N. Shiryaev. Singapore: World Scientific, 1996. To appear

[Mur2] Muraki, N.: Stochastic integration on the monotone Fock space. In preparation

[Oba] Obata, N.: White Noise Calculus and Fock Space. Lecture Notes in Mathematics 1577, BerlinHeidelberg-New York: Springer, 1994

[OhP] Ohya, M., Petz, D.: Quantum Entropy and Its Use. Berlin - Heidelberg - New York: Springer, 1993

[Par] Parthasarathy, K. R.: An Introduction to Quantum Stochastic Calculus. Basel - Boston - Berlin: Birkhaüser, 1992 
[Sch1] Schürmann, M.: Quantum $q$-white noise and a $q$-central limit theorem. Commun. Math. Phys. 140, 589-615(1991)

[Sch2] Schürmann, M.: White Noise on Bialgebras. Lecture Notes in Mathematics 1544, Berlin - Heidelberg - New York: Springer, 1993

[Seg] Segal, I. E.: Tensor algebras over Hilbert spaces I. Trans. Amer. Math. Soc. 81, 106-134(1956)

[Spe] Speicher, R.: A new example of 'independence' and 'white noise.' Prob. Th. Rel. Fields 84, 141-159 (1990)

[Voi] Voiculescu, D. V.: Symmetries of some reduced free product $C^{*}$-algebras. In: Operator Algebras and their Connections with Topology and Ergodic Theory.

Proceedings, Buşteni, Romania 1983, ed. by H. Araki, C. C. Moore, Ş. Strătilæ and D. Voiculescu. Lecture Notes in Mathematics 1132, Berlin - Heidelberg - New York: Springer, 1985, pp.556-588

[vW] Von Waldenfels, W.: An algebraic central limit theorem in the anticommuting case. Z. Wahr. Verw. Gebiete 42, 135-140(1978)

\section{Communicated by H. Araki}

Postscript. After revising the paper, the author was informed by Professor Y. G. Lu at the Random Walk Miniworkshop held in Nagoya University on 20-21 June 1996 that he had obtained similar results in the setting of his theory of interacting free Fock spaces, in: Y. G. Lu, "An interacting free Fock space and reciprocal semi-elliptical law". To appear in Me. Fun. Ana. Topo. and in: M. De Giosa and Y. G. Lu, "From quantum Bernoulli process to creation and annihilation operators on interacting q-Fock space", submitted to Nagoya Math. J. 